\title{
Trivium
}

Revue franco-allemande de sciences humaines et sociales - Deutsch-französische Zeitschrift für Geistesund Sozialwissenschaften

$17 \mid 2014$

Relire Marcel Mauss

\section{La réception de Mauss en langue allemande}

\section{Stephan Moebius et Frithjof Nungesser}

Traducteur : Julie Sentis

\section{OpenEdition}

\section{Journals}

Édition électronique

URL : http://journals.openedition.org/trivium/4828

ISSN : 1963-1820

Éditeur

Les éditions de la Maison des sciences de l'Homme

\section{Référence électronique}

Stephan Moebius et Frithjof Nungesser, «La réception de Mauss en langue allemande », Trivium [En ligne], 17 | 2014, mis en ligne le 30 septembre 2014, consulté le 08 septembre 2020. URL : http:// journals.openedition.org/trivium/4828

Ce document a été généré automatiquement le 8 septembre 2020

\section{(c) (i) (9)}

Les contenus des la revue Trivium sont mis à disposition selon les termes de la Licence Creative Commons Attribution - Pas d'Utilisation Commerciale - Pas de Modification 4.0 International. 


\title{
La réception de Mauss en langue allemande
}

\author{
Stephan Moebius et Frithjof Nungesser \\ Traduction : Julie Sentis
}

\section{NOTE DE L'AUTEUR}

Nous remercions Erhard Schüttpelz pour ses précieuses indications.

1 La réception de Marcel Mauss en langue allemande commençait déjà de son vivant. Ce fut en particulier le sociologue René König, si important pour la sociologie ouestallemande d'après-guerre, qui présenta les travaux de Mauss à un large public dès le début des années 1930 en Allemagne ${ }^{1}$. Invité par Richard Thurnwald à séjourner à Paris pour y travailler « Les nouveaux courants de la sociologie française contemporaine ", un article $^{2}$ qui paraît ensuite en 1931/1932 dans la revue de Thurnwald Völkerpsychologie und Soziologie (plus tard Sociologus), René König entre en contact avec l'école de Durkheim, et en particulier Marcel Mauss avec lequel «se nouèrent très vite d'étroites relations de travail ${ }^{3} »$. C'est ainsi que König appréhende l'école durkheimienne comme le lien étroit, si typique de la sociologie française, entre sociologie et ethnologie ${ }^{4}$. König désigne Mauss dans son article comme l'héritier le plus direct de Durkheim et « comme la tête de l'école de Durkheim ${ }^{5}$ ». König témoigne déjà par là, d'une large connaissance de l'école durkheimienne et de ses recherches.

2 Le travail de René König sur les durkheimiens va pourtant plus loin : à l'hiver 1932/1933 est conçu dans la petite bastide appartenant à ses parents dans le sud de la France le manuscrit : «La sociologie 'objective' de Emile Durkheim ». Encouragé entre autres par Alfred Vierkandt, Werner Sombart, Max Dessoir et Wolfgang Köhler, il espère avec cet écrit être habilité. Mais une habilitation sur le socialiste réformiste et juif Emile Durkheim n'est plus possible en 1933 en Allemagne : König prend la route de l'exil en 1937 vers Zürich ${ }^{6}$. Les durkheimiens cependant ne quittent pas les pensées du jeune sociologue. König publie après la seconde guerre mondiale une succession 
d'articles sur Durkheim et également sur Mauss, traduit les Règles de la méthode sociologique de Durkheim (qui paraissent ensuite en 1961). Il est pour l'essentiel responsable des publications des sociologues français ainsi que des comptes-rendus en langue allemande dans les années 1950 et $1960^{7}$. Aussi n'est-il pas étonnant qu'il s'entretienne plus fréquemment en 1960 avec Georges Gurvitch à propos de Mauss ${ }^{8}$.

3 Au cours de l'année 1972, König publie un article savant et instructif sur Marcel Mauss dans le Kölner Zeitschrift für Soziologie und Sozialpsychologie, que nous avons choisi pour le présent dossier9. A la différence de la réception et des raccourcis plus tardifs de la pensée de Marcel Mauss qui la réduisaient à l'Essai sur le don, König y traite plus précisément de l'œuvre de Mauss en son entier et en vient systématiquement au fond de sa pensée. Depuis le fait social total, l' « homme total » c'est-à-dire «complet », la théorie des civilisations en passant par la théorie des symboles sociaux jusqu'au « relationnisme structural » de Mauss et sa théorie de l'attente collective, König distille une suite de concepts sociologiques, qui, dans leur réception ultérieure en langue allemande, en particulier en sociologie, ont disparu. Pourquoi en est-il ainsi ? Un bref aperçu de la sociologie après 1945 permet de donner une réponse à cette question.

L'école durkheimienne est l'un des centres d'intérêt majeurs de König dans le champ sociologique de la République Fédérale d'Allemagne. Après 1945, elle est centrale pour la compréhension de la position scientifique et morale de König. Pendant l'aprèsguerre, jouer la carte de Durkheim n'était pourtant pas sans risque, a fortiori dans un environnement où l'école durkheimienne est peu connue ${ }^{10}$. Ainsi Adorno essaya-t-il en 1967 de faire d'une pierre deux coups en démontant dans son introduction à une collection d'articles de Durkheim Sociologie et philosophie, aussi bien Durkheim que König et - au temps de la querelle du positivisme -, ce que Adorno comprenait sous ce terme ${ }^{11}$. Adorno va même jusqu'à rejeter Durkheim, le socialiste réformiste et moraliste, du côté des idéologues fascistes ${ }^{12}$. König reconnaît immédiatement ce jeu de pouvoir au sein du champ sociologique et réagit aux « bavures hargneuses » de Adorno par une postface au Suicide dans laquelle il corrige toutes les accusations et autres erreurs d'interprétation ${ }^{13}$.

5 Malgré les efforts de René König réalisés afin de distinguer les mérites scientifiques propres à Mauss et bien que des écrits centraux fussent traduits en allemand à la fin des années 1960 et au milieu des années $1970^{14}$, la réception de Mauss reste jusque dans les années 1990 indubitablement dans l'ombre de Durkheim et de Claude Lévi-Strauss également. La célèbre autant qu'incontestée "Introduction à l'œuvre de Marcel Mauss » de Lévi-Strauss, qui précédait aussi bien la collection de texte français de l'année 1950 que sa traduction allemande, fut à cet égard significative. L'image qui y est donnée de Mauss, tel finalement un génial prédécesseur du structuralisme quoique de façon trop peu systématique, marqua durablement la réception de Mauss ${ }^{15}$. C'est la raison pour laquelle Mauss ne trouve qu'un écho seulement encore dans les reconstructions de l'anthropologie structurale de Lévi-Strauss.

6 Au plus tard à partir de 1990 se développa ensuite une réception plus large de Mauss, qui reste pourtant souvent réduite à l'Essai sur le don ${ }^{16}$. En effet, on rencontre toujours ces deux lectures bien rôdées - Mauss en tant que "protostructuraliste ${ }^{17}$ » ou bien fidèle adepte de Durkheim ${ }^{18}$-, mais des lectures plus différenciées émergent maintenant également. Une importance substantielle est attribuée à Mauss en ethnologie ou en anthropologie culturelle en particulier. "A bon droit ", comme l'écrit Petermann $^{19}$, on pourrait dire que Mauss - bien qu'il n'ait «jamais été lui-même 
ethnologue au sens académique du terme » - aurait "fondé l'ethnologie française ». Bien plus tôt déjà, Girtler le compte également - d'ailleurs influencé en cela par René König - parmi les «fondateurs de l'anthropologie culturelle ${ }^{20}$ ». En général, les références aux travaux de Mauss comme la caractérisation de son œuvre et ses conséquences prennent significativement plus de place dans les ouvrages généraux ou d'introduction à l'ethnologie en comparaison à la littérature sociologique correspondante. En partie, le don occupe ici aussi une place centrale ${ }^{21}$ mais l'on y trouve aussi des présentations plus détaillées ${ }^{22}$. Dans certains domaines de recherche en ethnologie, Mauss est un - sinon le - point de départ classique. Cela vaut pour les thématiques centrales de l'anthropologie des religions et de l'économie comme la magie, le sacrifice, le don ou l'échange ${ }^{23}$. Mais cela est également vrai pour des recherches qui se consacrent aux représentations du «temps » et de l' «identité »; des articles de Mauss sur les Inuits et le concept de personne sont sur ce point les références classiques ${ }^{24}$.

7 Relativement à la réception de Mauss dans le champ des sciences des religions, on peut évoquer en première ligne le spécialiste leipzigois Heinz Mürmel, qui n'a pas seulement présenté d'instructifs travaux sur la théorie de la magie chez Mauss ou sur le rôle de Mauss en tant que sociologue des religions ${ }^{25}$ mais a aussi fait connaître l'Essai sur le don en son entier comme une œuvre relevant des sciences des religions ${ }^{26}$. Du côté des sciences historiques, Mauss est consulté dans le cadre de recherches sur le mécénat bourgeois ou sa théorie du don rapprochée des théories de la reconnaissance ${ }^{27}$. La discussion relative au rapport entre don et reconnaissance - déclenchée par les lectures de Mauss faites par Georges Bataille, Jacques Derrida, Jean-Luc Marion ou encore Marcel Hénaff ${ }^{28}$ - eut pour effet que Mauss fût reçu en philosophie, en particulier lorsqu'il est question de reconnaissance ${ }^{29}$. Cela va jusqu'à rapprocher étroitement Hegel et Mauss $^{30}$. En sciences de gestion et dans les recherches en économie, on s'est également inspiré du don maussien et avec lui, une morale de la réciprocité centrale pour les relations de coopération (inter-)organisationnelles a été placée au cœur de la recherche ${ }^{31}$; la réciprocité et le don n'en ont pas pour autant été confondus avec l'échange. Ici se révèle une perception du don qui avant cela avait été soulignée en particulier dans les lectures de l'Essai sur le don faites par les nouvelles sciences et théories de la culture: la théorie du don dans cette perspective, comme Erhard Schüttpelz ${ }^{32}$ et Iris Därmann ${ }^{33}$ l'ont étudié dans d'instructives contributions, a été surtout détachée des théories de l'échange ou de la réciprocité qui y sont fréquemment liées. On l'a portée à connaissance comme une théorie des "mélanges » (en français dans le texte) entre choses et personnes ou bien encore comme une théorie de l'obsession ${ }^{34}$, comme une " théorie de l'appropriation croisée, du prendre réciproque et de l'être-pris des choses et des personnes ${ }^{35} »$. Ces préoccupations des sciences de la culture pour le don ont ouvert à bien des égards de nouvelles perspective sur le travail de Mauss, qui - pour ce que nous en savons - n'ont toutefois pas encore été prises en considération dans la réception de Mauss en langue française ${ }^{36}$.

8 Les différents courants de sa réception en sociologie se repèrent de la façon suivante : dans les années 1990 environ, Helmut Berking ${ }^{37}$ commence à s'inspirer de Mauss en tant que sociologue, ce en quoi - à la différence de König - il ne considère plus Mauss dans sa globalité mais comprend au contraire le don au sens d'un " présent ». Wolfgang Eßbach $^{38}$ et Axel Paul ${ }^{39}$ prennent un point de vue complètement différent et s'intéressent à la parenté entre don et vengeance. Le rattachement de Mauss à une théorie de la réciprocité est également significatif, comme y procèdent par exemple 
Frank Adloff ${ }^{40}$ dans plusieurs publications. Les implications de l'œuvre de Marcel Mauss en sociologie des émotions ont été en dernier lieu précisées ${ }^{41}$. Un autre courant s'intéresse au travail de Mauss du point de vue de l'histoire de la sociologie pour le présenter comme un classique de la sociologie qui a son caractère propre ${ }^{42}$ et un intellectuel engagé ${ }^{43}$. En outre, des efforts sont faits pour intensifier les échanges franco-allemands sur Mauss et examiner la fécondité heuristique de l'Essai sur le don pour la théorie des sciences sociales et de la culture ${ }^{44}$.

Les plus récents efforts concentrés sur la réception de Mauss concernent les parutions des travaux que l'on ne trouvait pas jusqu'alors en allemand - que ce soit le Manuel d'ethnographi $e^{45}$ ou les Écrits sur la sociologie des religions ${ }^{46}$. C'est ainsi qu'ont été révélés au public de langue allemande les mérites scientifiques propres à Mauss ${ }^{47}$, qui ne le distinguent pas seulement comme le gendre de Durkheim ou le théoricien poststructuraliste du symbolique mais bien comme un inspirateur de l'école durkheimienne, un regénérateur de l'ethnologie et un sociologue majeur des religions.

\section{BIBLIOGRAPHIE}

Adloff, F. (2005) : «Die Reziprozität der Gesellschaft - Zum Paradigma der Gabe in der Moderne », in : Corsten, M. et al. (éd.) : Die Gerechtigkeit der Gesellschaft, Wiesbaden : VS, p. 25-51.

Adloff, F. (2007) : « Marcel Mauss - Durkheimien oder eigenständiger Klassiker der französischen Soziologie ? », Berliner Journal für Soziologie, 2, p. 231-251.

Adloff, F. / Mau, S. (éd.) (2005) : Vom Geben und Nehmen. Zur Soziologie der Reziprozität, Francfortsur-le-Main / New York : Campus.

Adloff, F. / Papilloud, C. (éd.) (2008) : Alain Caillé : Anthropologie der Gabe, Francfort-sur-le-Main / New York : Campus.

Adorno, T. W. (1967) : « Einleitung », in : Émile Durkheim : Soziologie und Philosophie, Francfortsur-le-Main : Suhrkamp, p. 7-44.

Bedorf, T. (éd.) (2009) : Gabe und Anerkennung. Journal Phänomenologie, 31.

Bedorf, T. (2010) : Verkennende Anerkennung, Berlin : Suhrkamp.

Berking, H. (1996) : Schenken. Zur Anthropologie des Gebens, Francfort-sur-le-Main / New York : Campus.

Bogards, R. (éd.) (2010) : Texte zur Kulturtheorie und Kulturwissenschaft, Stuttgart : Reclam.

Därmann, I. (2005) : Fremde Monde der Vernunft. Die ethnologische Provokation der Philosophie, Munich : Fink.

Därmann, I. (2010) : Theorien der Gabe zur Einführung, Hambourg : Junius.

Elwert, G. (1991) : « Gabe, Reziprozität und Warentausch. Überlegungen zu einigen Ausdrücken und Begriffen », in : Berg, E. et al. (éd.) : Ethnologie im Widerstreit. Kontroversen über Macht, Geschäft, Geschlecht in fremden Kulturen. Festschrift für Lorenz G. Löffler, Munich : Trickster. 
Eßbach, W. (1999) : «Gabe und Rache. Zur Anthropologie der Gegenseitigkeit », in : TreuschDieter, G. et al. (éd.) : Schuld, Tübingen : Konkursbuchverlag, p. 11-20.

Girtler, R. (1979) : Kulturanthropologie. Entwicklungslinien, Paradigmata, Methoden, Munich : dtv. Girtler, R. (1989) : Die feinen Leute. Von der vornehmen Art, durchs Leben zu gehen, Linz : Veritas. Göbel, M. / Ortmann, G. / Weber, C. (2007) : « Reziprozität. Kooperation zwischen Nutzen und Pflicht », in : Schreyögg, G. / Sydow, J. (éd.) : Kooperation und Konkurrenz. Managementforschung 17, Wiesbaden : Gabler / VS, p. 161-205.

Gurvitch, G. (2006) : Écrits allemands III, éd. par C. Papilloud et C. Rol, Paris : L’Harmattan.

Hahn, H. P. (2013) : Ethnologie. Eine Einführung, Berlin : Suhrkamp.

Hénaff, M. (2002) : Le prix de la verité. Le don, l'argent, la philosophie, Paris : Seuil.

Hesse, K. (1987) : « Tausch », in : Streck (1987a), p. 224-227.

Honneth, A. (2010) : « Vom Gabentausch zur sozialen Anerkennung. Unstimmigkeiten in der Sozialtheorie von Marcel Hénaff », WestEnd. Neue Zeitschrift für Sozialforschung, 7(1), p. 99-110.

Huebner, D. R. (2013) : «Wilhelm Jerusalem's sociology of knowledge in the dialogue of ideas ", Journal of Classical Sociology, 13(4), p. 430-459.

Keller, T. (2004) : « Mauss et l'ethnologie allemande », L’Année sociologique, 54, p. 33-36.

Keller, T. (2006) : « Cassirer und Mauss. Ein Geistergespräch über Totemismus », in : Moebius, S. / Papilloud, C. (éd.) : Gift - Marcel Mauss' Kulturtheorie der Gabe, Wiesbaden : VS, p. 107-122.

König, R. (1972) : « Marcel Mauss (1872-1950) », Kölner Zeitschrift für Soziologie und Sozialpsychologie, 24, p. 633-657 (cf. traduction française dans ce numéro de Trivium).

König, R. (1976) : « Emile Durkheim. Der Soziologe als Moralist », in : Kaesler, D. (éd.) : Klassiker des soziologischen Denkens, Bd. 1 : Von Comte bis Durkheim, Munich, 1976, p. 312-364.

König, R. (1978) : Émile Durkheim zur Diskussion. Jenseits von Dogmatismus und Skepsis, Munich / Vienne : Hanser.

König, R. (1984) : Leben im Widerspruch. Versuch einer intellektuellen Autobiographie, Francfort-sur-leMain et al. : Ullstein.

König, R. (2013) : Schriften aus letzter Hand, Bd. 8 : Émile Durkheim. Zur Bestimmung der französischen Soziologie in Deutschland, éd. et postface par C. Albrecht, Wiesbaden : VS.

Köpping, K.-P. (2002) : " “Meissle deine Maske”. Autonome Person, gespaltenes Selbst oder Modul ? Ethnologische Anmerkungen zu Marcel Mauss », in : Köpping, K.-P. et al. (éd.) : Die autonome Person - Eine europäische Erfindung ?, Munich : Fink, p. 45-66.

Kohl, K.-H. (2002) : Ethnologie. Die Wissenschaft vom kulturell Fremden, 2. Aufl., Munich : Beck.

Krämer, H. L. (2000) : « Die Durkheimianer Marcel Mauss (1872-1950) und Maurice Halbwachs (1877-1945) », in : Kaesler, D. (éd.) : Klassiker des soziologischen Denkens, Bd. 1 : Von Auguste Comte bis Norbert Elias, Munich : Beck, p. 252-277.

Kramer, F. (1987a) : «Identität », in : Streck (1987a), p. 89-92.

Kramer, F. (1987b) : « Zeit », in : Streck (1987a), p. 261-264.

Mauss, M. (2013a) : Handbuch der Ethnographie, hg. v. I. Därmann u. K. Mahlke, Paderborn : Fink. Mauss, M. (2013b) : Schriften zur Religionssoziologie, hg. v. S. Moebius, F. Nungesser u. C. Papilloud, Berlin : Suhrkamp. 
Moebius, S. (2006a) : Marcel Mauss, Konstanz : UVK.

Moebius, S. (2006b) : « Intellektuelle Kritik und Soziologie. Die politischen Schriften von Marcel Mauss ", in : Moebius, S. / Schäfer, G. (éd.) : Soziologie als Gesellschaftskritik. Wider den Verlust einer aktuellen Tradition. Festschrift für Lothar Peter, Hamburg : VSA, p. 142-160.

Moebius, S. (2006c) : « Die Gabe - ein neues Paradigma der Soziologie ? Eine kritische Betrachtung der M.A.U.S.S.-Gruppe », Berliner Journal für Soziologie, 16(3), p. 355-370.

Moebius, S. (2009) : « Die elementaren (Fremd-)Erfahrungen der Gabe. Sozialtheoretische Implikationen der Kultursoziologie der Besessenheit von Marcel Mauss und des "radikalen Durkheimismus" des Collège de Sociologie », Berliner Journal für Soziologie, 19(1), p. 104-126.

Moebius, S. (2013) : « Die Religionssoziologie von Marcel Mauss », Zeitschrift für Religionswissenschaft, 19(1-2), p. 86-147.

Moebius, S. (2014) : « Die Kölner Schule », in : Fischer, J. / Moebius, S. (éd.) : Soziologische Denkschulen. Zur Archäologie der bundesrepublikanischen Soziologie (sous presse).

Moebius, S. / Peter, L. (2004) : Französische Soziologie der Gegenwart, Constance : UTB / UVK.

Moebius, S. / Papilloud, C : (éd.) (2006) : Gift - Marcel Mauss' Kulturtheorie der Gabe, Wiesbaden : VS.

Moebius, S. /Nungesser, F. (2013) : " "La filiation est directe" - Der Einfluss von Marcel Mauss auf das Werk von Claude Lévi-Strauss ", European Journal of Sociology / Archives Européennes de Sociologie / Europäisches Archiv für Soziologie, 54(2), p. 231-263 (cf. traduction française dans ce numéro de Trivium).

Mürmel, H. (1985) : Das Magieverständnis von Marcel Mauss, unveröff. Dissertation, Universität Leipzig.

Mürmel, H. (1991) : « Frazer oder Mauss : Bemerkungen zu Magiekonzeptionen », Zeitschrift für Missionswissenschaft und Religionswissenschaft, 75, p. 147-154.

Mürmel, H. (1997) : « Marcel Mauss », in : Michaels, A. (éd.) : Klassiker der Religionswissenschaft, Munich : WBG, p. 211-221.

Mürmel, H. (2000) : « Einige Bemerkungen zu Marcel Mauss’ Essai sur le don als religionswissenschaftliches Werk », in : Flasche, R. et al. (éd.) : Religionswissenschaft in Konsequenz. Beiträge im Anschluß an Impulse von Kurt Rudolph, Münster : Lit, p. 175-184.

Nungesser, F. / Moebius, S. (2012) : « Marcel Mauss : "Der obligatorische Ausdruck von Gefühlen (australische orale Bestattungsrituale)" und weitere Schriften », in : Senge, K. / Schützeichel, R. (éd.) : Hauptwerke der Emotionssoziologie, Wiesbaden : VS, p. 226-235.

Paul, A. (1996) : FremdWorte : Etappen der strukturalen Anthropologie, Francfort-sur-le-Main : Campus.

Paul, A. (2005) : « Die Rache und das Rätsel der Gabe », Leviathan. Berliner Zeitschrift für Sozialwissenschaft, 33(2), p. 240-256.

Peter, L. (2013) : « Dialektik der Gesellschaft oder “conscience collective” ? Zur Kritik Theodor W. Adornos an Émile Durkheim », in : Bogusz, T. / Delitz, H. (Hg) : Émile Durkheim. Soziologie Ethnologie - Philosophie, Francfort-sur-le-Main /New York : Campus, p. 73-94.

Petermann, W. (2004) : Die Geschichte der Ethnologie, Wuppertal : Peter Hammer Verlag.

Pielhoff, S. (2007a) : «Stifter und Anstifter. Vermittler zwischen “Zivilgesellschaft”, Kommune und Staat im Kaiserreich«, Geschichte und Gesellschaft : Schenken, Stiften, Spenden, 33, p. 10-45. 
Pielhoff, S. (2007b) : « Schwierige Geschenke. Anerkennungskonflikte zwischen Avantgardekünstlern, Vermittlern und Mäzenen im wilhelminischen Kaiserreich », KulturPoetik. Journal for Cultural Poetics, 2, p. 179-198.

Quadflieg, D. (2010a) : « Asymmetrische Reziprozität. Über das Verhältnis von Gabe und Anerkennung bei Marcel Hénaff », WestEnd. Neue Zeitschrift für Sozialforschung, 7(1), p. 111-122.

Quadflieg, D. (2010b) : « Der Geist der Sache. Hegels Jenaer "Philosophie des Geistes” und das Gabe-Theorem von Marcel Mauss ", in : Arndt, A. et al. (éd.) : Geist ? Hegel-Jahrbuch, 2010, Berlin : Akademie, p. 89-94.

Quadflieg, D. (2011) : « Die Unmöglichkeit der Gabe anerkennen. Anerkennung und Zeitlichkeit bei Hénaff, Ricoeur und Derrida », in : Hetzel, A. et al. (éd.) : Alterität und Anerkennung, BadenBaden : Nomos, p. 77-91.

Ritter, H. (1990) : « The Ethnological Revolution : On Marcel Mauss », Comparative Civilizations Review, 22, p. 1-18.

Schüttpelz, E. (2005) : Die Moderne im Spiegel des Primitiven, Munich : Fink.

Stentzler, F. (1979) : Versuch über den Tausch. Zur Kritik des Strukturalismus, Berlin : Medusa.

Streck, B. (éd.) (1987a) : Wörterbuch der Ethnologie, Köln : DuMont.

Streck, B. (1987b) : « Magie », in : Streck (1987a), p. 132-135.

Streck, B. (1987c) : « Opfer », in : Streck (1987a), p. 157-160.

\section{NOTES}

1. Sur le rôle central de René König pour la sociologie et « l'esprit de l'époque » de la République fédérale comme «l'école de Cologne » de König, voir Moebius (2014). Avant König, cela a été Wilhelm Jerusalem qui utilisa pour sa sociologie des sciences les connaissances de Mauss, Emile Durkheim et Henri Hubert ; ceux-ci présentèrent derechef les travaux de Jerusalem au public français. Voir sur ce point Huebner (2013), p. 441 sqq.

2. König (1978), p. 56-103 ; König (2013), p. 23-64.

3. König (1984), p. 97.

Voir la lettre de Mauss à König du 3 mai 1932 aux archives municipales de Cologne. Mauss se base - à côté de Malinowski, Franz Boas ou Robert Hertz - sur Thurnwald de nouveau dans son célèbre Essai sur le don.

4. Voir König (1984), p. 91. Sur la sociologie française, voir Moebius / Peter (2004).

5. König (1978), p. 60.

6. Pour la première fois en 1975, König publie son habilitation passée à la fin des années 30 à Zurich, une Critique de la sociologie historico-existentialiste. Contribution à la fondation d'une sociologie objective. La dernière partie est le manuscrit évoqué sur Durkheim.

7. Voir König (2013).

8. Voir Gurvitch (2006), p. 187 sqq. ; König (1984), p. 99 et p. 101 sq.

9. König (1972) ; voir aussi König (2013), p. 150-180.

10. Le peu d'attention porté à l'école de Durkheim après les Première et Seconde Guerre mondiale contraste fort avec l'intérêt des durkheimiens à l'ethnologie, la philosophie, les sciences des religions et la sociologie de langue allemande. Voir sur cette question Keller (2004), (2006).

11. Voir Peter (2013).

12. Adorno (1967), p. 15. 
13. Voir la « Postface au Suicide» dans : König (1978), p. 208-238, ici p. 215 sqq. Voir aussi König (1976), p. 328.

14. En 1968 paraît l'Essai sur le don traduit en allemand par Eva Moldenhauer. En 1974 et 1975 suit la traduction allemande par Henning Ritter de la collection de texte en deux tomes Sociologie et Anthropologie. Ce fut à l'origine en 1950, l'année du décès de Mauss, que parurent en un même ouvrage les écrits centraux de l'auteur. A côté des travaux sur le don qui y figurent également, se trouvent la "Théorie de la magie » de Hubert et Mauss comme l'étude exhaustive "Sur les variations saisonnières des sociétés eskimos" (cette dernière fut cependant ajoutée pour la première fois en 1966). De plus, quelques courts travaux sur les rapports entre sociologie et psychologie, sur les représentations culturelles de la mort, sur les «techniques des corps » comme sur le concept de personne y sont également intégrés.

15. Sur la production de cette image de Mauss, voir l'article de Moebius et Nungesser traduit ici (ou dans sa version originale en allemand parue en 2013). Sur l'influence de Mauss sur LeviStrauss, voir aussi Paul (1996), p. 62 sqq.

16. L'autre article repris de nouveau était relatif aux « techniques des corps » sur lequel il attirait l'attention en particulier à travers la sociologie du corps et les Gender Studies alors en développement, thématisant la construction sociales des réalités corporelles, ce qui est maintenant également repris dans les sciences de la culture (voir Bogards [2010], p. 111-119).

17. Voir Ritter (1990).

18. Krämer (2000).

19. Peterman (2004), p. 815.

20. Girtler (1979), p. 50.

21. Voir par exemple Kohl (2002), p. 89, 99, 142 ; Hahn (2013), p.137 sqq.

22. Voir Girtler (1979), p. 32 sq., 108 sqq., 223 sqq., 254 sqq. et Petermann (2004), p. 811 sqq.

Le sociologue et anthropologue culturel autrichien Roland Girtler inscrit fortement sa représentation de la recherche en anthropologie culturelle en général dans la tradition de Mauss (voir par exemple Girtler [1979], p. 33 et 45 ; [1989], p. 16 sq.). Il utilise aussi ses concepts dans la recherche empirique et relie ainsi d'une certaine façon les réflexions de Mauss et Thorstein Veblen (voir Girtler [1989]) dans son étude Les gens biens.

23. Voir par exemple Hesse (1987) ; Streck (1987b), (1987c) ; Elwert (1991).

24. Voir Kramer (1987a), (1987b) ; Köpping (2002).

25. Voir Mürmel (1985), (1991), (1997).

26. Mürmel (2000).

27. Voir Pielhoff (2007a), (2007b).

28. Hénaff (2002).

29. Voir Bedorf (2009), (2010); Honneth (2010); Quadflieg (2010a), (2011).

30. Quadflieg (2010b).

31. Voir Göbel/ Ortmann / Weber (2007).

32. Schüttpelz (2005).

33. Därmann (2005), (2010).

34. Därmann (2005), p. 102 sqq. ; Schüttpelz (2005), p. 179 sqq.

35. Schüttpelz (2005), p. 185.

Une lecture - toutefois davantage encore orientée sur l'échange - de l'Essai sur le don comme théorie des mélanges ainsi qu'indice d'une théorie du don cannibaliste de «l'incorporation des autres » se trouve chez Stentzler (1979), p. 43 sqq. et p. 64.

36. Pour cette raison, le texte de Erhard Schüttpelz a été inclus dans ce dossier.

37. Berking (1996).

38. Eßbach (1999).

39. Paul (2005).

40. Adloff (2005), Adloff / Mau (2005). 
41. Nungesser / Moebius (2012).

42. Moebius (2006a); Adloff (2007).

43. Voir Moebius (2006b).

44. Voir Moebius / Papilloud (2006) ; Moebius (2006c) ; Adloff / Papilloud (2008) ; Moebius (2009).

45. Mauss (2013a).

46. Mauss (2013b).

47. Voir Moebius (2013).

INDEX

Schlüsselwörter : Marcel Mauss, Rezeption, Wissenschaftsgeschichte, Soziologiegeschichte, Geschichte der Ethnologie

Mots-clés : Marcel Mauss, réception, histoire des sciences, histoire de la sociologie, histoire de l'ethnologie

\section{AUTEURS}

\section{STEPHAN MOEBIUS}

Stephan Moebius est professeur de théorie sociologique et d'histoire des idées à l'Université Karl Franz de Graz. Pour plus d'informations, voir la notice suivante.

\section{FRITHJOF NUNGESSER}

Frithjof Nungesser est assistant à l'Institut de sociologie de l'Université Karl Franz de Graz. Pour plus d'informations, voir la notice suivante. 